\title{
Álvaro Moreno Leoni \& Agustín Moreno, Historiografía Moderna y Mundo Antiguo (1850- 1970), Córdoba, Tinta Libre, 2018, 189 p. ISBN 978-987-708-295-1.
}

Felipe Montanares Piña, Universidad de Concepción. ORCID: https://orcid.org/0000-00017627-5682

El presente volumen coeditado por Álvaro Moreno Leoni y Agustín Moreno, es la publicación de las presentaciones realizadas en el IV Workshop Nacional de Historia Antigua y Estudios Clásicos (Historiografía Moderna y Mundo Antiguo 1850-1970), que tuvo cita el año 2016 en la ciudad de Córdoba, Argentina. Los investigadores que presentaron sus trabajos fueron los participantes del proyecto de investigación Memoria, conceptos y escritura de la historia: aproximaciones a la historiografía moderna sobre el mundo antiguo (1850-1970) avalado por la Secretaría de Ciencia y Tecnología de la Universidad Nacional de Córdoba, que contó con la participación de investigadores de Brasil y España.

El escrito se encuentra dividido en seis capítulos que en su conjunto logran demarcar un claro seguimiento en la evolución de las interpretaciones historiográficas sobre la antigüedad en el mundo moderno, esto queda claro al realizar una revisión de la bibliografía consultada por los autores, actualizada y debidamente relacionada a los problemas que se han de tratar a lo largo del Volumen.

El primer capítulo "Historiografía Moderna y mundo antiguo clásico, siglos XIX-XX: a modo de introducción" escrito por los editores del libro, nos presentan los problemas de la interpretación de los estudios clásicos en el mundo moderno, estableciendo un viaje de ida y retorno entre las fuentes, los intelectuales modernos con sus interpretaciones, y claramente los estudios contemporáneos que pueden vislumbrar en esta relación respuestas a problemas de nuestra propia contemporaneidad. Partiendo en la fundación de la universidad de Berlín en 1810, los autores dedican atención al desarrollo de las ciencias de la antigüedad de la mano de autores como Johann J. Winckelmann (1717-1768), Friedrich A. Wolf (1759-1824) y Christian Gottlob Heyne (1759-1824). Estos autores fueron el comienzo de un camino que se desarrolló desde Alemania a Francia, Italia e Inglaterra, centrando su atención en las nociones de Imperio, civilización y libertad, todo determinado en la relaciones del mundo griego y romano, a través de sus respectivos procesos de aculturación. Finalmente, los editores se remiten a comentar las ideas de autores británicos como William W. Tarn (1860-1957) o Francis Haverfield (1860-1919) y la influencia de autores de importancia en los estudios de la antigüedad como Theodor Mommsen (1817-1903) en sus trabajos.

El segundo capítulo es de Juan Pablo Alfaro, "Profesor Balsdon: De la renovación crítica a la nostalgia post-imperialista". El autor nos invita a reflexionar en torno a las ideas del historiador 
John Percy Vivian Dacre Balsdon (1901-1977), separando en los tres apartados del capítulo, tres momentos de la vida del autor, relacionando así sus trabajos sobre el mundo antiguo con la experiencia personal y sus interpretaciones de la realidad en las primeras décadas del siglo XX. La primera de estas etapas nos presenta una historiografía que estaba al servicio del imperialismo, donde prima un paternalismo occidental británico, que se refleja en la relación de las ideas de civilización y barbarie, en un mundo en el cual los británicos tenían como deber la tarea de civilizar. En este contexto la comprensión del proceso de romanización se remitía a este rol civilizatorio de los británicos en el mundo. Las nuevas perspectivas del siglo XX en conjunto con la adopción de un método cualitativo en la crítica de las fuentes, donde toma importancia una reflexión desde la hermenéutica, nos invitan a contemplar en los trabajos de Balsdon una nueva interpretación de los acontecimientos, debido principalmente a los cambios generacionales desde el fin de la primera guerra mundial al periodo de descolonización. Su obra The emperor Gaius es un hito en la renovación crítica del periodo, analizando elementos como la crueldad y la locura. Esta obra, publicada en el periodo anterior a la Segunda Guerra Mundial, manifestó elementos que se relacionan con el periodo post-imperialista británico. Balsdon se posiciona como un intelectual en un periodo de cambios, que mira levemente con nostalgia el paternalismo cívico en un mundo que enfrentó fuertes movimientos con el desarrollo de la Segunda Guerra Mundial.

El tercer capítulo se titula "Un estudio sobre la recepción de Polibio por Gaetano de Sanctis" escrito por Breno Battistin Sebastiani. Como señala el nombre del apartado la investigación, se dirige al análisis de la obra de Gaetano de Sanctis (1870-1957) tomando en consideración algunas ideas desarrolladas por Frank Walbank en un ensayo llamado "Polybius through the eyes of Gaetano de Sanctis". ${ }^{1}$ El autor establece una relación entre la censura de las elecciones políticas de Polibio con la postura personal de Gaetano de Sanctis contra Mussolini. Posteriormente Battistin se enfoca en la revisión el apéndice a los capítulos II y III del volumen III.1 de la Storia dei Romani, (1917), estableciendo que las preocupaciones del historiador italiano se han de dirigir hacia dos puntos: en primer lugar la articulación de la narrativa hasta el proceso editorial, y por otra parte de la religiosidad de Polibio. Se concluye el apartado enfatizando en la importancia de la percepción y práctica del oficio del historiador llevado por De Sanctis.

El cuarto apartado se titula "Historia y comparativismo: Sir Moses Finley y el concepto de imperio en el mundo antiguo" escrito por Diego Alexander Olivera. Este capítulo se orienta a desarrollar la obra de Moses Immanuel Finley (1912-1986), un autor ineludible para los especialistas en el mundo clásico. El autor divide la vida de Moses I. Finley en dos periodos:

\footnotetext{
${ }^{1}$ Articulo presente en la obra del mismo autor Walbank, F. Polybius, Rome and the Hellenistic World: Essays and Reflection, Cambridge, Cambridge University Press, 2002, pp. 310-321.
} 
desde 1927-1954, la etapa americana, y desde 1954-1986, la etapa británica. Olivera en el presente texto nos propone pensar en dos artículos publicados por Finley sobre el imperialismo en el Mundo mediterráneo antiguo. Realizando un análisis metodológico del proceso comparativo de Finley, observa que la relación entre propiedad de la tierra y la pertenencia cívica fue el motor del imperialismo en el mundo grecorromano. Podemos señalar que el autor realiza una deconstrucción del concepto de imperio, partiendo de lo particular en cada caso, para finalmente establecer las diferencias y relaciones aplicadas en el método comparativo utilizado por Finley.

El quinto capítulo se llama, "Entre el 'gobierno de la muchedumbre' y la 'dictadura del proletariado': la historiografía de la democracia ateniense frente al espejo de la revolución" escrito por Diego Paiaro. El artículo presenta una serie de problemas en torno al proceso de conceptualización de la democracia ateniense en el mundo moderno, estableciendo el recorrido de las tesis en torno a esta desde la revolución francesa a la reconfiguración por la revolución rusa, poniendo de manifiesto una interpretación del concepto de democracia en un contexto de revolución. El autor establece que, desde la antigüedad, en la misma ciudad ateniense comenzó la circulación de las ideas antidemocráticas. Para justificar esta afirmación, nos plantea que los escritos que nos han sido legados registran las ideas solo de un grupo social que no representó a la totalidad de la sociedad ateniense, tradición que al momento de la conquista por los macedonios ya se había establecido. En ese sentido surge la idea de un temor a las multitudes o el "Gobierno de la muchedumbre" al cual se le atribuye la decadencia de la democracia ateniense. Posteriormente nos presenta una discusión entre William Mitford (1744-1827) como un crítico de esta idea de la dependencia y la ociosidad de los atenienses, con la postura de George Grote (1794-1871), quien al contrario nos plantea una revalorización de la democracia antigua, poniendo las bases para un rescate de esta interpretación como un modelo. Resulta interesante que este análisis permite el uso del pasado en el presente, a través del estudio e interpretación de conceptos como democracia, que establecen un impacto en su propio contexto, lo que nos brinda un marco para comprender su propia situación.

Finalmente, el sexto capítulo que cierra este volumen se llama "Una mirada sobre el trabajo de otros: Función y uso de la reseña en la obra de Arnaldo Momigliano" en las palabras de Cesar Sierra, quien realizó un análisis de las 145 reseñas realizadas por Arnaldo Momigliano (19081987). El autor nos propone para ello dos perspectivas de análisis: la primera desde lo cuantitativo, realizando una revisión a través de la cantidad de publicaciones y las revistas en las cuales fueron publicadas, y la segunda desde la hermenéutica, atendiendo a la capacidad del historiador y su habilidad para desarrollar el contenido en las reseñas. En primera instancia se presenta una clara biografía del autor dividida en una sencilla distribución a lo largo de los acontecimientos de su experiencia personal. De igual manera se desarrolla un apartado de los antagonismos académicos que desenvolvió a lo largo de su carrera, con Piero Treves (1911- 
1992), Ronald Syme (1903-1989) y Luciano Canfora (1942-). El uso de gráficos y tablas son de utilidad para visualizar el análisis cuantitativo de sus reseñas y su distribución en las revistas.

El presente volumen cumple con su objetivo de establecer y comprender la relación entre la antigüedad y la edad moderna, pero sin duda, también nos permite reconocer el proceso que deben hacer los historiadores para poder comprender el pasado. Así, desde la reflexión en torno al proceso de pensamiento y aplicación metodológica de los autores seleccionados, podemos reconocer las herramientas que utilizan los historiadores para comprender su propio presente, proceso que no es ajeno inclusive a nuestra propia contemporaneidad, ni nuestras propias herramientas de trabajo.

Con una diagramación muy amable a la vista, debemos destacar que cada una de las citas de este texto se encuentra traducida al español, facilitando su lectura y siendo de gran ayuda para los interesados que no han de manejar otros idiomas. Cada uno de los estudios es una invitación a reflexionar nuestros propios procesos de representación historica, nuestra propia metodología y sin duda un importante apoyo teórico en el planteamiento de nuevas hipotesis para comprender la antigüedad desde nuestro presente. 